\title{
BMJ Open Differences in terms of presentation and outcomes between patients with lung cancer as opposed to other solid organ cancer after infection with SARS-CoV-2: protocol for a systematic review
}

Haisheng Chen, Jing Shi, Wenna Shi, Hengwei Xu, Cunxian Duan, Qing Fan, Yanhong Wang, Hui Li

To cite: Chen $\mathrm{H}$, Shi J, Shi W, et al. Differences in terms of presentation and outcomes between patients with lung cancer as opposed to other solid organ cancer after infection with SARS-CoV-2: protocol for a systematic review. BMJ Open 2020;10:e041790. doi:10.1136/ bmjopen-2020-041790

- Prepublication history and additional material for this paper are available online. To view these files, please visit the journal online (http://dx.doi. org/10.1136/bmjopen-2020041790).

Received 17 June 2020 Revised 24 September 2020 Accepted 23 October 2020

Check for updates

(C) Author(s) (or their employer(s)) 2020. Re-use permitted under CC BY-NC. No commercial re-use. See rights and permissions. Published by BMJ.

Department of Pharmacy, Shandong Cancer Hospital and Institute, Shandong First Medical University and Shandong Academy of Medical Sciences, Jinan, Shandong, China

Correspondence to Hui Li; 874223972@qq.com

\section{ABSTRACT}

Introduction Scholars believe that COVID-19 can be particularly lethal for patients with cancer. Some studies found that COVID-19 appears to be more lethal in patients with lung cancer than in other cancer patients. In order to take appropriate measures to balance a delay in lung cancer treatment against the risk for a potential COVID-19 exposure, we first need to know whether patients with lung cancer have special risks. We aim to conduct a systematic review and meta-analysis to examine differences in terms of presentation and outcomes between patients with lung cancer as opposed to other solid organ cancer after infection with SARSCoV-2.

Methods and analysis A comprehensive search of published original research studies will be performed in Embase, MEDLINE, Web of Science, WangFangData, CQVIP, COMPENDEX and CNKI. The medRxiv preprint server will also be searched for applicable studies (grey literature). Original research studies will be included if they include patients with: (A) laboratory-confirmed SARS-CoV-2 infection and (B) confirmed solid cancer, and (C) measurable clinical presentation or outcome, such as mortality rate, intensive care unit admission rate, incidence of pneumonia. One author will conduct the electronic database searches, two authors will independently screen studies, two will extract data and two will assess study quality. If $\left.\right|^{2}$ exceeds $60 \%$ for the pooled analysis, we will explore sources of heterogeneity in subgroups of studies. We will use fixed-effect, random-effects or mixed-effects models to estimate the relative risk or $\mathrm{OR}$. If the data reporting allows, a subgroup analysis between non-small cell lung cancer and small cell lung cancer patients will be performed.

Ethics and dissemination The proposed study will not collect individual-level data and, therefore, does not require ethical approval. We will submit our findings to a peer-reviewed scientific journal and will disseminate results through presentations at international scientific conferences.

PROSPERO registration number CRD42020190118.
Strengths and limitations of this study

- This systematic review will be the first to systematically review studies that have investigated differences in terms of presentation and outcomes of COVID-19 in patients with lung cancer as opposed to other solid organ cancer patients.

- If appropriate, we will perform a meta-analysis to examine differences in terms of presentation and outcomes between patients with lung cancer as opposed to other solid organ cancer after infection with SARS-CoV- 2 by calculating and comparing mortality rate, intensive care unit admission rate and incidence of pneumonia.

- Comparable studies, with data disaggregated into lung cancer/other solid organ cancer, may be limited.

- We are only including studies published in the English and Chinese language, which may lead to language bias.

\section{INTRODUCTION}

Infection with SARS-CoV-2, resulting in COVID-19, can lead to acute respiratory distress syndrome requiring admission to an intensive care unit (ICU), and sometimes death, in a subset of patients. New cases of the COVID-19 continue to rise worldwide. So far, we know that individuals $\geq 60$ years of age and/or those with a suppressed immune system are particularly vulnerable to COVID19 , although how these risks apply to patients with cancer remains unclear. ${ }^{1}$ Scholars such as Liang $e t a t^{2}$ found that COVID-19 can be more lethal in patients with cancer than individuals without cancer. A recent meta-analysis of 32 studies involving 46499 patients (1776 patients with cancer) suggested that cancer is associated with worse clinical outcomes among patients with COVID-19. ${ }^{3}$ 
While all types of malignancies seem to be associated with high COVID-19 prevalence, morbidity and mortality, lung cancer represents a specific scenario of cumulative risk factors for COVID-19 complications, including older age, significant respiratory comorbidities, smokingrelated lung damage, as well as the unavoidable addition of treatment-related immune impairment or suppression. ${ }^{4}$ National Health Service England warned that certain groups are particularly vulnerable to serious illness if they become infected with SARS-CoV-2. These groups include individuals who are undergoing active chemotherapy or radical radiotherapy for lung cancer and patients with cancers of the blood or bone marrow. ${ }^{5}$ Recently, the TERAVOLT registry revealed that patients with thoracic malignancies are at increased risk of prolonged hospitalisation and mortality from COVID-19. Of the 200 patients, $67 \%$ met the criteria for ICU admission and 33\% died. While in the meta-analysis mentioned above, the average mortality rate of 1428 cancer patients with COVID-19 from eight studies was 13.5\%. ${ }^{3}$

During the COVID-19 pandemic, the European Society for Medical Oncology (ESMO) clinical recommendations for lung cancer management are a guide to ensure and maintain high-quality standards for patients with lung cancer. ${ }^{6}$ It provides a structured proposal for the management of lung cancer, comprising three levels of priorities, defined according to the criteria of the Cancer Care Ontario, Huntsman Cancer Institute and Magnitude of Clinical Benefit Scale. Unfortunately, to date, robust data are lacking to guide adjustments to standard of care in patients with lung cancer.

In order to take appropriate measures to balance a delay in lung cancer treatment against the risk for a potential SARS-CoV-2 exposure, we first need to know if patients with lung cancer are at special risks. ${ }^{7}$ This current review, to the best of our knowledge, will be the first to systematically review studies that have investigated differences in terms of presentation and outcomes between patients with lung cancer as opposed to other solid organ cancer after infection with SARS-CoV-2.

\section{OBJECTIVE}

The objective is to conduct a systematic review and metaanalysis to examine differences in terms of presentation and outcomes between patients with lung cancer as opposed to other solid organ cancer after infection with SARS-CoV-2.

\section{REVIEW QUESTIONS}

This systematic review will primarily address the following research question:

1. Are the mortality rate and ICU admission rate of lung cancer patients with COVID-19 higher than that of other solid organ cancer patients with COVID-19?

2. Is the incidence of pneumonia in lung cancer patients with COVID-19 higher than that in other solid organ cancer patients with COVID-19?

\section{METHODS AND ANALYSIS}

\section{Protocol registration and review reporting}

The protocol outlines our strategy to conduct a systematic review on differences in terms of presentation and outcomes between patients with lung cancer as opposed to other solid organ cancer after infection with SARS-CoV-2 informed by the Preferred Reporting Items for Systematic Reviews and Meta-Analyses (PRISMA) and the Meta-analysis of Observational Studies in Epidemiology statements. ${ }^{89}$ We will adopt the four-phase PRISMA flow diagram. This study protocol has been developed in line with the Preferred Reporting Items for Systematic Review and Meta-Analysis Protocols (PRISMA-P) statement. ${ }^{10} 11$ This systematic review has been registered with the International Prospective Register of Systematic Reviews (PROSPERO). ${ }^{12}$

\section{Eligibility criteria}

We will use our research questions to identify key concepts and determine the Population, Exposure, Comparator for the exposure and desired Outcome (PECO). These components will be used to determine which studies meet the selection criteria. The following is an outline of the inclusion criteria based on the PECO concept:

\section{Inclusion criteria}

Study design: there will be no restriction on the type of study design eligible for inclusion, as the current data on COVID-19 and cancer is limited.

Population: patients of any age or gender from any country.

Exposure: patients with a laboratory-confirmed SARS-CoV-2 infection and confirmed lung cancer.

Comparator: patients with a laboratory-confirmed SARS-CoV-2 infection and confirmed solid organ cancer other than lung cancer.

Outcome: measurable clinical presentation or outcome, such as mortality rate, ICU admission rate and incidence of pneumonia.

\section{Exclusion criteria}

Published conference abstracts.

Published study protocols.

Published review articles.

Patients in whom SARS-CoV-2 infection has not been laboratory confirmed.

Patients whose solid organ cancer not been confirmed. Studies performed on animal models.

\section{Patient and public involvement}

Patients and/or the public were not involved in the design, or conduct, or reporting, or dissemination plans of this research.

\section{INFORMATION SOURCES}

Searches will be performed in electronic databases including Embase, MEDLINE, Web of Science, WangFangData, CQVIP, COMPENDEX and CNKI. The searches will 
be restricted to English and Chinese language papers. All peer-reviewed original research articles published before October 2020 will be included. Given the rapidly evolving nature of this topic, the medRxiv preprint server will also be searched for applicable studies (grey literature). The reference lists of all relevant study manuscripts will be reviewed manually to identify any additional studies of interest.

\section{SEARCH STRATEGY}

The search strategy will be guided by PRISMA-P. A search strategy will be structured to identify relevant studies for this systematic review. The search strategy will include controlled vocabulary and Medical Subject Headings to identify the concepts 'cancer' and 'COVID-19'. See the online supplemental table one for an example of the MEDLINE search strategy.

\section{STUDY RECORDS}

The search strategy described above will be used to identify abstracts for screening. All abstracts obtained will be imported to the Endnote software to speed up article selection and remove duplicates. Titles and abstracts of the studies will be independently screened by two reviewers. Abstracts that do not mention lung cancer will not be discarded at this stage.

\section{SCREENING}

Two investigators will independently screen the titles and abstracts of the articles identified from the literature search. The PRISMA for abstracts checklist will be used as a guideline to execute the inclusion criteria of the review. Full text of eligible abstracts will be reviewed using the standardised PRISMA 2009 checklist. The full text of the remaining studies will be retrieved and reviewed independently by two investigators. An impartial third investigator will be consulted if the two investigators are unable to reach a consensus on the inclusion of studies.

\section{DATA EXTRACTION AND DATA ITEMS}

Data extraction will be performed independently by two investigators. An Excel spreadsheet will be used to record the following data extracted from eligible studies; the first author's name, year of publication, country name, study design, sample size, study population characteristics (eg, age, gender and smoking), cancer type, COVID-19 infection characteristics, presenting symptoms, other comorbidities, pneumonia incidence, morbidity rate and ICU admission rate. Data extraction will be done by one reviewer and checked by a second reviewer. Differences will be resolved by discussion and consensus.
QUALITY ASSESSMENT AND RISK OF BIAS IN INDIVIDUAL STUDIES

Eligible studies will be critically appraised by two investigators to assess the quality and the risk of bias of the studies included in the review. Newcastle-Ottawa quality assessment scale will be used to determine the quality of nonrandomised studies. ${ }^{13}$ Publication bias will be assessed via funnel plots. The quality appraisal will be appropriately adjusted for grey literature. Any discordant outcome will be resolved through discussion with a third reviewer.

\section{DATA SYNTHESIS, ANALYSIS AND ASSESSMENT OF HETEROGENEITY}

If there are significant differences in the study designs and methodologies, we will provide a narrative summary of the findings. Quantitative data will be summarised for studies with comparable methodologies and presented in tables and forest plots reporting weighted summary statistics through Revman software. We will report a summary measure of the individual studies to obtain a pooled estimates of the OR for cross-sectional or case-control studies and Relative Risk (RRs) for cohort studies.

Heterogeneity across the included studies will be investigated and quantified. The Cochrane $Q$ statistic will be used to assess heterogeneity across studies included in meta-analysis. The inconsistency index $\left(\mathrm{I}^{2}\right)$ will be used as quantified measure of statistical heterogeneity. According to the Cochrane Handbook, we suppose a moderate level of heterogeneity between studies for $\mathrm{I}^{2}$ values ranging from $30 \%$ to $60 \%$. If $\mathrm{I}^{2}$ exceeds $60 \%$ for the pooled analysis, we will explore sources of heterogeneity in subgroups of studies. We will use fixed-effect, random-effects or mixed-effects models to estimate the relative risk or OR.

\section{ANALYSIS OF SUBGROUPS OR SUBSETS}

If the data reporting allows, a subgroup analysis between non-small cell lung cancer and small cell lung cancer patients will be performed. In addition, if it is appropriate to conduct a meta-analysis, a quality sensitivity analysis will also be conducted by excluding studies at high risk of bias. Furthermore, we will undertake subgroup analyses to investigate whether covariates exist and to examine heterogeneity in our outcome. Analyses will be performed for subgroups stratified by patient age, sex, smoking status, comorbidities, country and study risk of bias (low vs high) if the sample volume is enough.

\section{ETHICS AND DISSEMINATION}

The proposed study will not collect individual-level data and, therefore, does not require ethical approval. The results of this systematic review will provide the most up to date literature synthesis on differences in terms of presentation and outcomes between patients with lung cancer as opposed to other solid organ cancer after infection with SARS-CoV-2. Results will be disseminated through 
peer-reviewed publications and presentations at relevant national and international conferences.

\section{AMENDMENTS}

In the event of protocol amendments, we will provide the date of each amendment, describe the change and give the rationale for it.

Contributors $\mathrm{HL}$ conceived the idea. The protocol was developed by all authors. JS performed the search strategy and design the data extraction form. WS and $\mathrm{HX}$ will contribute to screening manuscripts. $\mathrm{HC}$ and $\mathrm{CD}$ will extract data from individual studies. QF and YW assess study quality. HL will act as a third reviewer in case of discrepancy. $\mathrm{HL}$ and $\mathrm{HC}$ redacted the original draft of the protocol. All authors reviewed and contributed to subsequent drafts and read and approved the final draft.

Funding $\mathrm{HL}$ is supported by Shandong Traditional Chinese Medicine science and Technology Development Project (2019-0532). WS is supported by the Shandong Provincial Key Research Development Program (2018GSF118175) and the Medicine and Health Science Technology Development Program of Shandong Province (2017WSA18034). QF is supported by the Youth Foundation Program of Shandong Academy of Medical Sciences (2018-27).

Competing interests None declared.

Patient consent for publication Not required.

Provenance and peer review Not commissioned; externally peer reviewed.

Supplemental material This content has been supplied by the author(s). It has not been vetted by BMJ Publishing Group Limited (BMJ) and may not have been peer-reviewed. Any opinions or recommendations discussed are solely those of the author(s) and are not endorsed by BMJ. BMJ disclaims all liability and responsibility arising from any reliance placed on the content. Where the content includes any translated material, BMJ does not warrant the accuracy and reliability of the translations (including but not limited to local regulations, clinical guidelines, terminology, drug names and drug dosages), and is not responsible for any error and/or omissions arising from translation and adaptation or otherwise.

Open access This is an open access article distributed in accordance with the Creative Commons Attribution Non Commercial (CC BY-NC 4.0) license, which permits others to distribute, remix, adapt, build upon this work non-commercially, and license their derivative works on different terms, provided the original work is properly cited, appropriate credit is given, any changes made indicated, and the use is non-commercial. See: http://creativecommons.org/licenses/by-nc/4.0/.

\section{ORCID iD}

Hui Li http://orcid.org/0000-0001-5583-5329

\section{REFERENCES}

1 Sidaway P. COVID-19 and cancer: what we know so far. Nat Rev Clin Oncol 2020;17:336.

2 Liang W, Guan W, Chen R, et al. Cancer patients in SARSCoV-2 infection: a nationwide analysis in China. Lancet Oncol 2020;21:335-7.

3 Giannakoulis VG, Papoutsi E, Siempos II. Effect of cancer on clinical outcomes of patients with COVID-19: a meta-analysis of patient data. JCO Glob Oncol 2020;6:799-808.

4 Passaro A, Peters S, Mok TSK, et al. Testing for COVID-19 in lung cancer patients. Ann Oncol 2020;31:832-4.

5 Burki TK. Cancer guidelines during the COVID-19 pandemic. Lancet Oncol 2020;21:629-30.

6 Passaro A, Addeo A, Von Garnier C, et al. ESMO management and treatment adapted recommendations in the COVID-19 era: lung cancer. ESMO Open 2020;5:e000820.

7 Kutikov A, Weinberg DS, Edelman MJ, et al. A war on two fronts: cancer care in the time of COVID-19. Ann Intern Med 2020;172:756-8.

8 Moher D, Altman DG, Liberati A, et al. PRISMA statement. Epidemiology 2011;22:128.

9 Stroup DF, Berlin JA, Morton SC, et al. Meta-Analysis of observational studies in epidemiology: a proposal for reporting. meta-analysis of observational studies in epidemiology (moose) group. JAMA 2000;283:2008-12.

10 Moher D, Shamseer L, Clarke M, et al. Preferred reporting items for systematic review and meta-analysis protocols (PRISMA-P) 2015 statement. Syst Rev 2015;4:1.

11 Shamseer L, Moher D, Clarke M, et al. Preferred reporting items for systematic review and meta-analysis protocols (PRISMA-P) 2015: elaboration and explanation. BMJ 2015;350:g7647.

$12 \mathrm{Li} \mathrm{H}$, Chen HS, Shi J. COVID-19 in lung cancer patients and other solid cancer patients: a systematic review examining differences in outcome and presentation, 2020. Available: https://wwwcrdyorkacuk/ prospero/display_recordphp?ID=CRD42020190118

13 Wells GA, Peterson J. The Newcastle-Ottawa scale (NOS) for assessing the quality of nonrandomised studies inmeta-analyses, 2014. Available: http://wwwohrica/programs/clinical_epidemiology/ oxfordasp 